\title{
APROXIMACIÓN AL ESTUDIO DE LAS NIEBLAS EN EL VALLE MEDIO DEL DUERO
}

\author{
Carlos Morales Rodríguez* \\ Ma Teresa Ortega Villazán*
}

\begin{abstract}
RESUMEN
Se realiza un estudio detallado de las nieblas en el valle medio del Duero, a fin de conocer su dinámica, frecuencia, distribución espacial y, sobre todo, se pretende analizar de forma pormenorizada los tipos de tiempo a los que se asocian, puesto que no siempre son las situaciones de marcada estabilidad sus causantes. Ante todo se resaltan los principales factores que contribuyen a su génesis, derivados tanto el relieve como la dinámica atmosférica, y se reflexiona sobre sus efectos y consecuencias.
\end{abstract}

\section{RESUMÉ}

On réalise une étude minutiense des brouillards dans la vallée centrale du Douro, afin de connaître leur dymanique, leur fréquence, leur distribution spatiale et, surtout, on prétend analyser en détail les types de temps aux quels ils s'associent, étant donné que les situations de stabilité marquée ne sont pas toujours la cause. On met surtout en relief les facteurs principaux qui contribuent à leur genèse, dérivés du relief ainsi que de la dynamique atmosphérique, et on réfléchit sur leurs effets et leurs conséquences.

Uno de los efectos meteorológicos más característicos a lo largo del valle del Duero durante el período invernal es la aparición de nieblas. Éstas, bien sean matinales, de escasa duración, o persistentes, crean uno de los ambientes climáticos más característicos de este valle en buena parte del invierno y, logran definir un rasgo propio del clima de esta cuenca. No obstante, pese a la habitual presencia de este meteoro año tras año y, la gran percepción que de él tienen los castellanos, ha sido un tema apenas tratado en los estudios climáticos, quizá por la dificultad que entraña el registro de este tipo de datos y por las escasas conclusiones que se puedan aportar.

Esta importante laguna y su habitual presencia durante los inviernos, nos ha animado a su análisis aún siendo conscientes de que los resultados obtenidos hayan de tomarse como

* Profesores del Departamento de Geografía de la Universidad de Valladolid. 
una primera aproximación, dejando abiertas las vías a futuras investigaciones relacionadas con este tema.

\section{Objetivos y metodología}

Nuestro objetivo principal es caracterizar las nieblas en el valle medio del Duero, concretamente en el transepto Soria-Valladolid, si bien de forma más precisa entre esta última ciudad y Almazán, puesto que a partir de las altas planicies sorianas, y como es lógico, los procesos neblinosos pierden entidad (Fig. 1). No obstante, el hecho de que uno de los autores tenga su centro de trabajo en Soria, le obliga a constantes desplazamientos diarios a lo largo de la carretera que sigue este valle, nos ha llevado a contabilizar también las de este núcleo. Así se ha podido no sólo comprobar in situ los rasgos de este meteoro, sino poder seguir la evolución de las nieblas durante muchos días, diversas horas y distintos observatorios, lo que nos ha facilitado crear nuestra propia base de datos, importante complemento de la información aportada por el Centro Meteorológico Zonal del Duero.

Los problemas para una buena caracterización de este meteoro son importantes principalmente por el tipo de dato que se maneja. En este caso la red de observatorios empleada no es tan parca ni mal distribuida como suele ser habitual, contándose sólo con aquéllos que ofrecían una información más fiable. Así el estudio se ha realizado a partir de 14 observatorios, siendo la mayoría termométricos y con unas series de observación en general continuas (Figura 1 y Cuadro I).

La noción de niebla es lábil al responder a la apreciación personal de visibilidad del observador, con lo que la subjetividad o falta de experiencia entraña inconvenientes al hacer el registro y dificulta posibles comparaciones entre distintos observatorios. Otro inconveniente es que las anotaciones se limitan a la toma del meteoro, independientemente de su intensidad, desarrollo..., así como su no observación durante la noche (momento más probable por el mayor enfriamiento del aire).

Cuadro I

LOCALIZACIÓN DE LOS OBSERVATORIOS Y PERÍODO DE OBSERVACIÓN

\begin{tabular}{|c|c|c|c|c|c|c|c|c|c|c|}
\hline & \multicolumn{2}{|c|}{ CARÁCTER } & \multicolumn{2}{|c|}{$\mathbf{N}^{0}$ AÑOS } & \multicolumn{2}{|c|}{ PERÍODO } & \multicolumn{2}{|c|}{ COORDENADAS } & \multicolumn{2}{|r|}{ FUENTE } \\
\hline Observatorios & $\mathrm{T}$ & $\mathbf{P}$ & $\mathbf{T}$ & $\mathbf{P}$ & $\mathrm{T}$ & $\mathbf{P}$ & Lat. & Long. & Alt. & \\
\hline Valladolid & + & + & 62 & 32 & $1931-92$ & $1961-92$ & $41-39$ & $1-04 \mathrm{~W}$ & 700 & CMZ DUERO \\
\hline Tudela & + & + & 48 & 31 & $1945-92$ & $1962-92$ & $41-35$ & $0-54 \mathrm{~W}$ & 704 & CMZ DUERO \\
\hline Sardón de Duero & + & + & 44 & 31 & $1948-92$ & $1961-92$ & $41-37$ & $0-44 \mathrm{~W}$ & 723 & CMZ DUERO \\
\hline Peñafiel & + & + & 12 & 15 & $1978-90$ & $1978-92$ & $41-36$ & $0-26 \mathrm{~W}$ & 756 & CMZ DUERO \\
\hline Roa & + & + & 9 & 27 & $1984-92$ & $1961-92$ & $41-40$ & $0-14 \mathrm{~W}$ & 750 & CMZ DUERO \\
\hline Castrillo de la Vega & & + & & 18 & & $1974-91$ & $41-39$ & $0-05 \mathrm{~W}$ & 805 & CMZ DUERO \\
\hline Aranda de Duero & + & + & 42 & 32 & $1951-92$ & $1961-92$ & $41-40$ & $0-00 \mathrm{~W}$ & 800 & CMZ DUERO \\
\hline La Vid & + & + & 48 & 30 & $1943-90$ & $1962-92$ & $41-38$ & $0-12 \mathrm{E}$ & 832 & CMZ DUERO \\
\hline S. Esteban de Gormaz & + & + & 61 & 32 & $1932-92$ & $1961-92$ & $41-34$ & $0-29 \mathrm{E}$ & 900 & CMZ DUERO \\
\hline El Burgo de Osma & + & + & 61 & 32 & $1932-92$ & $1961-92$ & $41-35$ & $0-37 \mathrm{E}$ & 895 & CMZ DUERO \\
\hline Gormaz & + & + & 6 & 6 & $1987-92$ & $1987-92$ & $41-30$ & $0-41 \mathrm{E}$ & 956 & CMZ DUERO \\
\hline Almazán & + & + & 60 & 32 & $1932-92$ & $1961-92$ & $41-29$ & 1-09E & 938 & CMZ DUERO \\
\hline Lubia & + & + & 0 & 32 & 1986 & $1961-92$ & $41-39$ & $1-11 \mathrm{E}$ & 1049 & CMZ DUERO \\
\hline Soria & + & + & 62 & 32 & $1931-92$ & $1961-92$ & $41-46$ & $1-13 \mathrm{E}$ & 1063 & CMZ DUERO \\
\hline
\end{tabular}




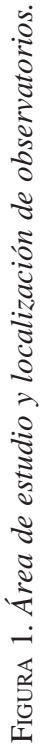


La carencia de una información más precisa nos ha impedido tratar aspectos tales como las horas de duración, el espesor de la nubosidad, su mantenimiento-disipación... deduciéndose muchas veces de modo indirecto. Así mismo nos ha llevado a exprimir al máximo la información disponible, recurriendo a datos diarios y no conformándonos con el empleo de los mensuales, válidos para caracterizaciones generales. Fundamental resultaba para relacionar los días de niebla con la dinámica atmosférica, y para poder realizar comparaciones entre distintos puntos del valle y ver su coincidencia o no en desarrollos.

Se ha tomado como referencia un período de cinco años, de 1988 a 1992, centrándonos exclusivamente en los inviernos, que hemos considerado oportuno extenderlos a cinco meses, de noviembre a marzo, pues climáticamente en estas tierras son prolongados, aparte de fríos, como ya ha sido suficientemente demostrado por distintos autores (CALONGE CANO, G. 1984; GARCÍA FERNÁNDEZ, J. 1986). En dicho período se ha analizado en primer lugar la dinámica atmosférica imperante, desglosando los distintos tipos de tiempo que han afectado a la Cuenca del Duero, para a continuación especificar día a día todas aquellas jornadas afectadas por niebla en los catorce observatorios. Así se ha pretendido:

- Individualizar áreas homogéneas en la percepción del meteoro.

- Relacionar las nieblas con una dinámica atmosférica específica a fin de comprobar qué tipos de tiempo son los responsables de su formación y cuáles son los más proclives a favorecerlas.

- Ver las posibles coincidencias en la aparición y desarrollo a lo largo del valle, para comprobar si se tratan de situaciones más o menos generalizadas o por el contrario se manifiestan como fenómenos aislados.

- Averiguar el promedio de días con niebla que a lo largo del invierno pueden afectar a este sector del valle del Duero.

- Analizar las características de las situaciones más persistentes para definir los rasgos propios del ambiente climático tan específico que crean.

- Distinguir entre distintos tipos de nieblas de acuerdo con su génesis, y realizar un primer intento de clasificación en el que se especifiquen la proporción con que se manifies$\tan$.

\section{El valle medio del Duero: una configuración morfológica muy propia para la formación de nieblas}

Si hubiera que buscar a un responsable principal para explicar la particular frecuencia que este meteoro tiene en el valle del Duero la respuesta sería el propio valle (Figura 2). En efecto, independientemente de la dinámica atmosférica a la que se asocien los estados neblinosos, siempre va a ser ésta en relación con la morfología del valle la que en última instancia los genere. Y es que el relieve, en este caso el progresivo encajamiento que experimenta el valle del Duero desde su cabecera, es el principal agente favorecedor del estancamiento de las masas de aire, preferentemente en situaciones de marcada estabilidad y con humedad ambiente. Desde los $1.063 \mathrm{~m}$ de Soria a los $698 \mathrm{~m}$ de Valladolid, el Duero se encaja más de $350 \mathrm{~m}$, desnivel bien expresivo al que se han de adaptar las masas de aire.

Pero no es sólo la morfología del valle del Duero la que contribuye a ello. La extensa cuenca creada por este río, rodeada de montañas en casi todo su alrededor, cuenta con una notable altitud media, en torno a los $900 \mathrm{~m}$. Factores que han condicionado el aislamiento del que participa frente a las situaciones de inestabilidad oceánicas, lo que se manifiesta no sólo en una menor cuantía de las precipitaciones, sino en unos mayores rasgos de continentalidad, con acusados contrastes térmicos estacionales e incluso diarios. Hechos que favorecen intensos procesos de irradiación. El Duero atraviesa esta cuenca en sentido zonal, 





salvo en su cabecera (desde su nacimiento hasta Almazán), y por su sector central, lugar hacia el que tienden a estancarse las masas de aire por ser un área de menor altitud.

Este valle, una vez que cruza las altas planicies sorianas, se encaja primero en las plataformas estructurales de la cuenca satélite del Burgo de Osma-Almazán y, más tarde, entre los páramos de la cuenca terciaria propiamente dicha (páramos de El Cerrato por el norte y páramos de Cuéllar-Campaspero por el sur), bien encauzado y abriendo progresivamente su lecho mayor hacia el oeste, ganando en amplitud la campiña (Figura 2). A lo largo del mismo las nieblas son frecuentes pero no en todos sus puntos alcanzan igual intensidad y duración, como veremos. Nuevamente la configuración geomorfológica es la responsable de crear diferencias a nivel local.

Los desniveles que se crean entre los páramos y las campiñas son lo suficientemente significativos como para permitir enfriamientos diferenciados y la movilidad del aire más frío y denso hacia el fondo del valle, donde se acumula. Entre las rígidas plataformas estructurales del Este y la ribera del Duero las diferencias altitudinales suelen ser inferiores al centenar de metros, pero desde la Tierra de Roa hacia Valladolid éstas se incrementan a los $150 \mathrm{~m}$, a veces más ${ }^{1}$. Así, el aire en contacto con los páramos calcáreos, se enfría más rápidamente y se desplaza hacia su base acumulándose y apelmazándose en los fondos de artesa de la campiña, a veces por largo tiempo.

Otro importante factor fomentador de las nieblas, aunque más secundario, es la existencia de abundantes cursos de agua a lo largo del valle. Junto al colector principal aparecen numerosos afluentes por ambas márgenes, fiel reflejo de la red jerarquizada de este río. Esta presencia de agua contribuye a humedecer las masas de aire por su base favoreciendo la condensación, aunque sea a nivel local. Siempre es más densa la niebla en las proximidades de una corriente fluvial.

En determinados sectores del valle este proceso toma protagonismo, como ocurre en el entorno de Valladolid, conocida entre otras cosas por lo habitual de este meteoro. Situada en la baja campiña del Pisuerga, en la confluencia con el Esgueva, que también la cruza, y entre las estribaciones de los páramos, por el oeste los Montes Torozos y al este los más digitados del Cerrato, los procesos de inversión durante el invierno son bien frecuentes. A ello también hay que unir la cada vez más creciente contaminación ambiental derivada tanto de la actividad industrial como del tráfico rodado. La emisión de partículas cada vez es mayor, actuando como núcleos de condensación en períodos de marcada estabilidad.

Así pues, partimos de unas condiciones geomorfológicas bien propicias para el desarrollo de nieblas, pasemos pues a analizarlas.

\section{Distribución espacial de los días de niebla}

Dentro del espacio analizado y, de acuerdo con el modo y frecuencia que tienen de distribuirse, en plena concordancia con las particularidades morfológicas del valle, se ha realizado una distinción en tres áreas: una se corresponde con la cuenca satélite del Burgo de Osma-Almanzán, otra con las campiñas del Duero medio y finalmente el valle del bajo Pisuerga (Figura 3).

A lo largo del valle el máximo de días de niebla invernales le corresponde a Valladolid, con una media superior a los 35 días, 38,4 para ser más exacto (cuadro II). Desde la ciudad

1 En la cuenca satélite del Burgo de Osma-Almazán la acumulación del aire frío y consecuentes procesos de inversión térmica se ven favorecidos tanto por la estrechez de este pasillo como por el desnivel que se crea entre ésta y las cordilleras que la delimitan por el norte (Cordillera Ibérica) y por el sur (Cordillera Central), dado que el contraste respecto a las plataformas estructurales que les son más próximas no es muy acusado. 
y remontando aguas al Duero se aprecia una progresiva disminución hasta Peñafiel donde se registran 15,4 días, algo menos de la mitad. No obstante, tanto en las campiñas del sur del Duero como en la Tierra de Roa las nieblas mantienen su importancia con valores por encima de los 25 días (Roa, 28,4 días). En el sector central, es decir, de Aranda de Duero a San Esteban de Gormaz, las nieblas anuales oscilan entre los 15 y 20 días, umbral que nuevamente se incrementa en la Cuenca satélite del Burgo de Osma-Almazán, alcanzándose en determinados enclaves, como en el Burgo de Osma (30,8 días), máximos importantes derivados muchas veces de aspectos locales.

En efecto, esta cuenca satélite constituye un enclave singular y especialmente proclive para el desarrollo de este tipo de meteoro. Se trata de un sector deprimido entre la Cordillera Ibérica y la Central, a modo de estrecho corredor $(35-45 \mathrm{~km})$ que se prolonga durante un centenar de kilómetros hasta Alhama de Aragón. El ser un espacio bien deprimido entre los relieves circundantes y su localización en la parte más oriental de la región castellana, en el centro peninsular y a sotavento de buen número de situaciones inestables y de los vientos dominantes, ha generado una importante continentalidad y, el que en situaciones de estabilidad los procesos de inversión térmica sean una constante.

En el caso concreto de El Burgo, su emplazamiento además contribuye a acrecentarlas. Rodeada de pequeños cerros que superan los $1.000 \mathrm{~m}$ de altitud, cúpulas de los pliegues cretácicos del sinclinorio que aquí se manifiesta, muy próxima a las estribaciones meridionales de la Sierra de Nafría, atravesada por el río Ucero y en sus proximidades la confluencia del Avión, son elementos favorecedores (Figura 3).

En sentido global, al valle le define una media significativa, pues salvo Peñafiel, Castrillo de la Vega y Aranda de Duero, siempre este número es superior a los 20 días. Quizá pueda sorprender que estos núcleos, y principalmente Peñafiel, alcancen valores tan reducidos, máxime cuando a pocos kilómetros la tendencia es contraria. Siempre queda la duda de la fiabilidad de los datos tomados y, en este caso, esta posibilidad se reafirma al haberse podido comprobar en los numerosos desplazamientos realizados, que normalmente si hay niebla en Valladolid esta se mantiene hasta Peñafiel. Es en la subida al páramo, en Fuentecén donde normalmente no aparecen, lo que justifica que también Castrillo de la Vega no las tenga y posiblemente a Aranda le cueste mantenerlas, que aunque en el valle, éste no se muestra tan encajado.

Otro aspecto que se ha comprobado es que si aparece niebla en el tramo de Fuentecén a Castrillo ésta se extiende hasta las proximidades de Soria, muchas veces hasta el Puerto de Villaciervos a pesar de la elevada altitud de esta planicie $(1.160 \mathrm{~m})$. Correspondería con las situaciones de niebla generalizada en el valle y por lo general persistentes.

Así pues, y como queda reflejado en la figura 3, los máximos de Valladolid, Roa y El Burgo parecen marcarnos los límites entre los tres sectores que antes apuntábamos. En todos ellos las nieblas son un elemento destacado, aunque convendría precisar varios aspectos:

- Que es en el bajo Pisuerga donde se registra el máximo de días de niebla de todo el espacio analizado, apreciándose desde Valladolid hacia Peñafiel una progresiva disminución, que nos aventuramos a decir que en la realidad posiblemente no sea tan marcada.

- Igualmente, al abandonar la cuenca satélite la disminución de las nieblas es importante. Véase la drástica reducción que experimentan desde Almazán a Soria al pasarse de los 21,5 a los 13 días (Cuadro II).

- En las campiñas del Duero medio parece apuntarse una mayor homogeneidad en registros disminuyendo su importancia en los páramos inmediatos.

Pero, si estos valores medios de invierno son indicativos de su importancia, más aún lo son las medias deducidas de los meses de noviembre, diciembre y enero, período en el 


\section{Cuadro II}

NÚMERO MEDIO DE DÍAS DE NIEBLA INVERNALES EN EL VALLE MEDIO DEL DUERO

\begin{tabular}{|lrrrrrrrrr|}
\hline Observatorios & Nov. & Dic. & Enero & Febr. & Marzo & TOTAL & \% $^{1}$ & Trimestre & $\%^{2}$ \\
\hline Valladolid & & & & & & & & & \\
Tudela de Duero & 8,7 & $\mathbf{1 2 , 3}$ & 12,0 & 3,8 & 1,6 & 38,4 & 25,4 & 33,0 & 35,9 \\
Sardón de Duero & 6,3 & $\mathbf{1 0 , 9}$ & 9,2 & 3,2 & 1,7 & 31,5 & 20,8 & 26,5 & 28,8 \\
Peñafiel & 5,6 & $\mathbf{8 , 1}$ & 7,2 & 2,4 & 1,3 & 24,6 & 16,3 & 20,9 & 22,6 \\
Roa & 4,1 & $\mathbf{5 , 1}$ & 4,7 & 1,0 & 0,5 & 15,4 & 10,2 & 13,9 & 15,1 \\
Castrillo de la Vega & 4,9 & $\mathbf{9 , 9}$ & 9,2 & 3,0 & 1,4 & 28,4 & 18,8 & 24,0 & 26,0 \\
Aranda de Duero & 3,6 & $\mathbf{5 , 8}$ & 3,3 & 1,0 & 0,2 & 13,9 & 9,2 & 12,7 & 13,8 \\
La Vid & 3,2 & $\mathbf{6 , 4}$ & 4,9 & 1,2 & 0,4 & 15,5 & 10,3 & 13,9 & 15,1 \\
San Esteban de Gormaz & 4,9 & $\mathbf{6 , 0}$ & 5,1 & 2,1 & 2,3 & 20,9 & 13,8 & 16,5 & 18,0 \\
Burgo de Osma & $\mathbf{7 , 3}$ & 5,7 & 2,0 & 1,1 & 20,8 & 13,8 & 17,8 & 19,4 \\
Gormaz & $\mathbf{5 , 0}$ & $\mathbf{9 , 2}$ & $\mathbf{7 , 4}$ & 4,6 & 2,7 & 30,8 & 20,4 & 23,5 & 25,6 \\
Almazán & 5,2 & $\mathbf{8 , 6}$ & $\mathbf{6 , 2}$ & 1,6 & 0,8 & 22,4 & 14,8 & 20,0 & 21,7 \\
Lubia & 4,9 & $\mathbf{6 , 7}$ & $\mathbf{7 , 1}$ & 2,0 & 0,7 & 21,5 & 14,2 & 18,7 & 20,4 \\
Soria & 3,8 & $\mathbf{4 , 4}$ & $\mathbf{4 , 9}$ & 1,4 & 0,5 & 15,0 & 9,2 & 12,0 & 13,0 \\
& 2,5 & $\mathbf{4 , 5}$ & 3,5 & 1,4 & 1,1 & 13,0 & 8,6 & 10,6 & 11,5 \\
\hline
\end{tabular}

Fuente: Centro Meteorológico Zonal del Duero. Datos diarios. Período 1961-92.

(1). Porcentaje respecto a los cinco meses invernales.

(2). Porcentaje respecto al trimestre noviembre-enero.

que realmente cobran todo su protagonismo (Cuadro II). En general, escasa es la diferencia respecto a los totales de invierno - lo que es indicativo de la tendencia a concentrarse en dicho trimestre-, siendo lo más llamativo los porcentajes que suponen respecto al total de días del mismo. De un modo global representan índices del 15 al $20 \%$, alcanzándose en los puntos más proclives porcentajes superiores al $25 \%$ de días del período, es decir, que aproximadamente un mes puede verse afectado por ellas. El caso más llamativo vuelve a ser Valladolid, donde suponen el 35,9 \%, unos 33 días de niebla de los 92 del trimestre.

\section{Variación y frecuencia de los días de niebla}

Tomando como base la información de los observatorios que disponen de series más dilatadas, se aprecia su escasa correlación en la evolución de los registros invernales. No hay coincidencia ni en cómputos totales ni en su distribución anual (Figura 4). Esta aleatoriedad, en principio desalentadora, tiene mucho que ver con la forma en que realmente se producen las nieblas en este valle.

En efecto, éstas cuando se producen no afectan siempre por igual a todos los observatorios pues al desarrollarse a base de bancos pueden aparecer en determinados puntos y en otros no hacerlo. También la heterogeneidad en los valores térmicos condiciona el modo de producirse dado que no siempre el frío ni la humedad se extienden de igual forma. A su vez, habría que considerar las diferencias morfológicas del valle a nivel local.

Al analizar las series de observación de cada estación se descubre otro aspecto de interés, la importante variabilidad interanual. Los coeficientes de variación calculados para las distintas series oscilan entre el $30-50 \%$, umbrales indicativos de esta irregularidad. Y en 


\begin{tabular}{|lrrrlll|}
\hline Observatorios & $\overline{\mathbf{X}}$ & $\begin{array}{r}\text { Sx } \\
(\%)\end{array}$ & $\begin{array}{c}\text { C.V. } \\
\text { Máx. }\end{array}$ & Dif. & Máx. Mens. (días) y Fecha \\
\hline Valladolid & 38,4 & 10,8 & 28,1 & 44 & 23 & Dic.(74) \\
Tudela de Duero & 31,5 & 9,6 & 31,5 & 42 & 21 & Dic.(88) \\
Sardón de Duero & 24,6 & 12,5 & 51,0 & 44 & 20 & Dic.(88), En.(90) \\
Roa & 28,4 & 10,0 & 35,2 & 40 & 25 & Dic.(74) \\
Aranda de Duero & 15,5 & 5,8 & 38,2 & 26 & 17 & Dic.(88) \\
La Vid & 20,9 & 10,5 & 50,2 & 38 & 21 & Dic.(74) \\
S. Esteban de Gormaz & 20,8 & 6,9 & 33,2 & 28 & 15 & Dic.(91) \\
El Burgo de Osma & 30,8 & 10,9 & 35,4 & 49 & 21 & En.(82) \\
Almazán & 21,5 & 9,2 & 42,8 & 43 & 19 & Dic.(74) \\
Lubia & 15,0 & 7,7 & 51,3 & 31 & 17 & En.(82) \\
Soria & 13,0 & 5,1 & 39,2 & 23 & 12 & Dic.(77) \\
\hline
\end{tabular}

Fuente: Centro Meteorológico Zonal del Duero. Datos diarios de la serie de que dispone cada observatorio.

$\overline{\mathbf{X}}$ : Media aritmética Sx: Desviación típica C.V.(\%): Coeficiente de variación.

este sentido el comportamiento a lo largo del valle es homogéneo, pues en todos los observatorios estos índices son elevados (cuadro III).

Otro elemento que ayuda a apreciar el grado de irregularidad en las nieblas es la diferencia máxima habida entre inviernos extremos. En todos es normal que entre los años con más nieblas y los de menos medien más de 25 días de diferencia, existiendo enclaves como Valladolid, Tudela, Sardón, Roa, El Burgo y Almazán donde superan los 40 días (44, $42,44,40,49$ y 43 respectivamente). La tendencia que se manifiesta es que en cada observatorio esta amplitud es superior a su registro medio y, por otra parte, donde hay mayor número de nieblas también los posibles intervalos de variación en los registros tienden a ser mayores. A modo de ejemplo, en Valladolid el invierno con mayor proporción de nieblas fue el de 1988-89 con 62 días y el que menos el de 1991-92 con 18 días, lo que supone 44 días de diferencia; en Soria el más neblinoso, 1977-78 con 27 jornadas y el que menos, 1981-82, tan sólo con 4, lo que se traduce en una amplitud de 23 días.

En lo que si suele haber coincidencia es en el mes con mayor proporción de nieblas. Este, salvo en Almazán y Lubia, siempre recae en diciembre, con valores que oscilan entre los 4,5 días de Soria a los 12,3 días de Valladolid (Cuadro II y Fig. 4). Nada extraño dada la particular tendencia que en este mes existe para el desarrollo de las situaciones anticiclónicas prolongadas.

Igualmente es diciembre junto con enero el período en el que se producen más registros de jornadas con nieblas, como se aprecia al disociarlas por umbrales y por meses (cuadro IV). Prácticamente en todos los observatorios, a excepción de Castrillo y Soria es normal que se superen los 10 días de niebla en el mes de noviembre, diciembre y enero, lo que varía como es lógico, es la frecuencia anual o proporción de años que en cada observatorio se ha superado ese registro. De todos ellos destaca Valladolid que en un período de 31 años el $41,9 \%$ de los mismos en noviembre ha superado ese umbral, el $71 \%$ en diciembre y el $64,5 \%$ en enero, lo que demuestra lo habitual de ese meteoro durante el invierno. Poco 
FIGURA 4 


\begin{tabular}{|c|c|c|c|c|c|c|c|c|c|c|c|c|c|c|c|c|}
\hline \multirow[b]{2}{*}{ Observatorios } & \multirow{2}{*}{$\begin{array}{c}\mathrm{N}^{0} \\
\text { Años }\end{array}$} & \multicolumn{5}{|c|}{$>10$ días } & \multicolumn{5}{|c|}{$>15$ días } & \multicolumn{5}{|c|}{$>20$ días } \\
\hline & & $\mathbf{N}$ & D & E & F & M & $\mathbf{N}$ & D & E & $\mathbf{F}$ & M & $\mathrm{N}$ & D & E & $\mathrm{F}$ & M \\
\hline Valladolid & 31 & 41,9 & 71,0 & 64,5 & 3,2 & - & 12,9 & 35,5 & 29,0 & - & - & - & 12,9 & 16,1 & - & - \\
\hline Tudela de Duero & 30 & 23,3 & 56,7 & 46,7 & 3,3 & - & 6,7 & 30,0 & 16,7 & - & - & - & 3,3 & - & - & 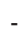 \\
\hline Sardón de Duero & 30 & 23,3 & 33,3 & 33,3 & - & - & 3,3 & 10,0 & 6,7 & - & - & - & 3,3 & 3,3 & - & - \\
\hline Peñafiel & 14 & 7,1 & 14,3 & 7,1 & - & - & - & - & - & - & - & - & - & - & - & - \\
\hline Roa & 26 & 7,7 & 50,0 & 50,0 & - & - & - & 19,2 & 7,7 & - & - & - & 7,7 & - & - & - \\
\hline Castrillo de la Vega & 17 & - & 17,6 & 5,9 & - & - & - & 5,9 & - & - & - & - & - & - & - & - \\
\hline Aranda de Duero & 31 & 3,2 & 22,6 & 12,9 & - & - & - & 6,5 & 3,2 & - & - & - & - & - & - & - \\
\hline La Vid & 29 & 6,9 & 27,6 & 13,8 & - & 3,4 & 3,4 & 6,9 & - & - & - & - & 3,4 & - & - & - \\
\hline S. Esteban de Gormaz & 31 & 9,7 & 32,3 & 6,5 & - & - & - & 3,2 & - & - & - & - & - & - & - & - \\
\hline El Burgo de Osma & 31 & 29,0 & 38,7 & 22,6 & 12,9 & - & - & 9,7 & 3,2 & 3,2 & - & - & - & 3,2 & - & - \\
\hline Almazán & 31 & 16,1 & 19,4 & 29,0 & - & - & - & 6,5 & 9,7 & - & - & - & - & - & - & - \\
\hline Lubia & 31 & 6,5 & 12,9 & 9,7 & - & - & - & 3,2 & 3,2 & - & - & - & - & - & - & - \\
\hline Soria & 31 & - & 12,9 & - & - & - & - & - & - & - & - & - & - & - & - & - \\
\hline
\end{tabular}

Fuente: Centro Meteorológico Zonal del Duero. Datos diarios.

frecuente es que se alcance este umbral de 10 días en febrero y marzo, bastante menos neblinosos que el resto.

La proporción de años que superan los 15 días de niebla al mes se reduce de forma importante, llegando a haber observatorios donde dicho umbral no se ha observado, como es el caso de Peñafiel o Soria. Este comportamiento se generaliza al elevarse el umbral hasta más de 20 días al mes. Únicamente en Valladolid, Tudela, Sardón, Roa, la Vid y El Burgo ha ocurrido en algún invierno, y siempre en los meses de diciembre y enero (Cuadro IV).

De todos los datos manejados el máximo mensual absoluto de días de niebla ha recaído en Roa en diciembre de 1974, mes en el que se registraron 25 días con niebla, siguiéndole de cerca Valladolid con 23 días.

\section{La distinta tipología de las nieblas y su relación con la dinámica atmosférica}

Para este análisis se ha empleado la información diaria de los Boletines Meteorológicos y de los datos de días de niebla del período 1988-1992, en los catorce observatorios ya especificados. En cada uno de ellos se han ido anotando los días que cada mes del invierno se han visto afectados por nieblas, a fin de relacionar las de todos ellos y verificar su coincidencia o no en el tiempo. De este modo, se puso de manifiesto que desde un punto de vista espacial existen nieblas que afectan de un modo generalizado al valle medio del Duero y, otras se producen mucho más aleatoriamente, más dispersas. Así mismo, hay nieblas que sólo duran algunas horas al cabo del día, mientras que otras se mantienen con persistencia, no sólo en una jornada sino en varias seguidas.

Normalmente las nieblas que dominan son las que mantienen durante varios días, con independencia de su intensidad y duración, por lo tanto, cabría definirlas con el calificativo 
de nieblas generalizadas. Por el contrario, aquellas más esparsas no suelen mantenerse más de un día por lo que se las puede caracterizar como nieblas aisladas o localizadas. Diferenciación que no puede entenderse de forma tajante pero si dominante.

Pero lo realmente importante de este comportamiento es conocer a qué génesis dinámica responden, de ahí que el siguiente paso haya sido señalar qué tipos de tiempo las motivan y ver cuáles lo hacen en mayor medida. Y en este sentido llama la atención el elevado número de situaciones que se han contabilizado para un período de cinco años $\mathrm{y}$, para la idea habitual que siempre se tiene de asociarlas de forma exclusiva con situaciones de marcada estabilidad atmosférica. Lógicamente las situaciones anticiclónicas son los principales artífices de su aparición pero no las únicas.

\section{Relaciones de la niebla con los distintos tipos de tiempo}

El análisis sinóptico se ha hecho con detenimiento y cierta escrupulosidad de ahí la variedad de tipos de tiempo anticiclónicos que aparecen en los cuadros elaborados (Cuadro V). Con esto lo que se pretende es destacar los diversos tipos de circulaciones atmosféricas capaces de originarlas, aunque muchas de ellas deparen efectos meteorológicos semejantes.

Como se advierte en el Cuadro V dentro del largo período invernal es el trimestre de noviembre-enero cuando los tipos de tiempo anticiclónicos cobran todo su protagonismo a la hora de generar nieblas. Por meses, en noviembre es el $72,3 \%$ de todas las posibles situaciones, en diciembre el 70,3\% y en enero $63,6 \%$. Tanto en febrero como en marzo estos porcentajes, aún siendo altos, disminuyen sobre todo en este último, donde ya suponen el 47,4 \%, y toman más importancia los tipos de tiempo inestables. Además mientras en aquél trimestre las nieblas son de mayor entidad por su prolongación temporal, al final del invierno se vuelven aisladas aún con los tipos de tiempo más favorables.

Esta forma de manifestarse la dinámica mensualmente también queda patente al centrarnos exclusivamente en las situaciones de estabilidad. De noviembre a enero son más relevantes a la hora de producir nieblas los tipos anticiclónicos claros, es decir, las crestas Tm amplias, las que se disponen en bloqueo en omega o las cuñas del suroeste, que aquellos que participan de los influjos de situaciones de inestabilidad (crestas Tm con circulación zonal o con vaguada Pm al Norte o al Este o situaciones de bloqueo difluente). Todo lo contrario ocurre en febrero y marzo donde son estos últimos tipos los que las producen en mayor medida. Este comportamiento es normal y en última instancia guarda estrecha relación con la evolución atmosférica a nivel global, pues la primavera astronómica siempre ha sido mucho más inestable que el invierno, al menos en el interior de esta región de Castilla-León.

\section{a) Las nieblas con situaciones anticiclónicas}

Las nieblas resultantes de situaciones de estabilidad se deben bien a procesos de irradiación nocturna o bien a procesos de advección-irradiación. Distinción que ya ha sido apuntada tanto por Calonge Cano, G. como por García Fernández, J. a propósito de las nieblas en Valladolid y en Castilla-León respectivamente. Quizá pueda sorprender que aire tan cálido como el tropical, dada su procedencia, sea capaz de generar nieblas intensas y prolongadas manteniendo bajas temperaturas durante varios días. Algo impensable en otros muchos puntos de España, pero en este valle, por la morfología de esta cuenca, el enfriamiento nocturno y las inversiones térmicas son muy habituales.

Estos tipos de tiempo cuando originan nieblas lo hacen durante todos o casi todos los 


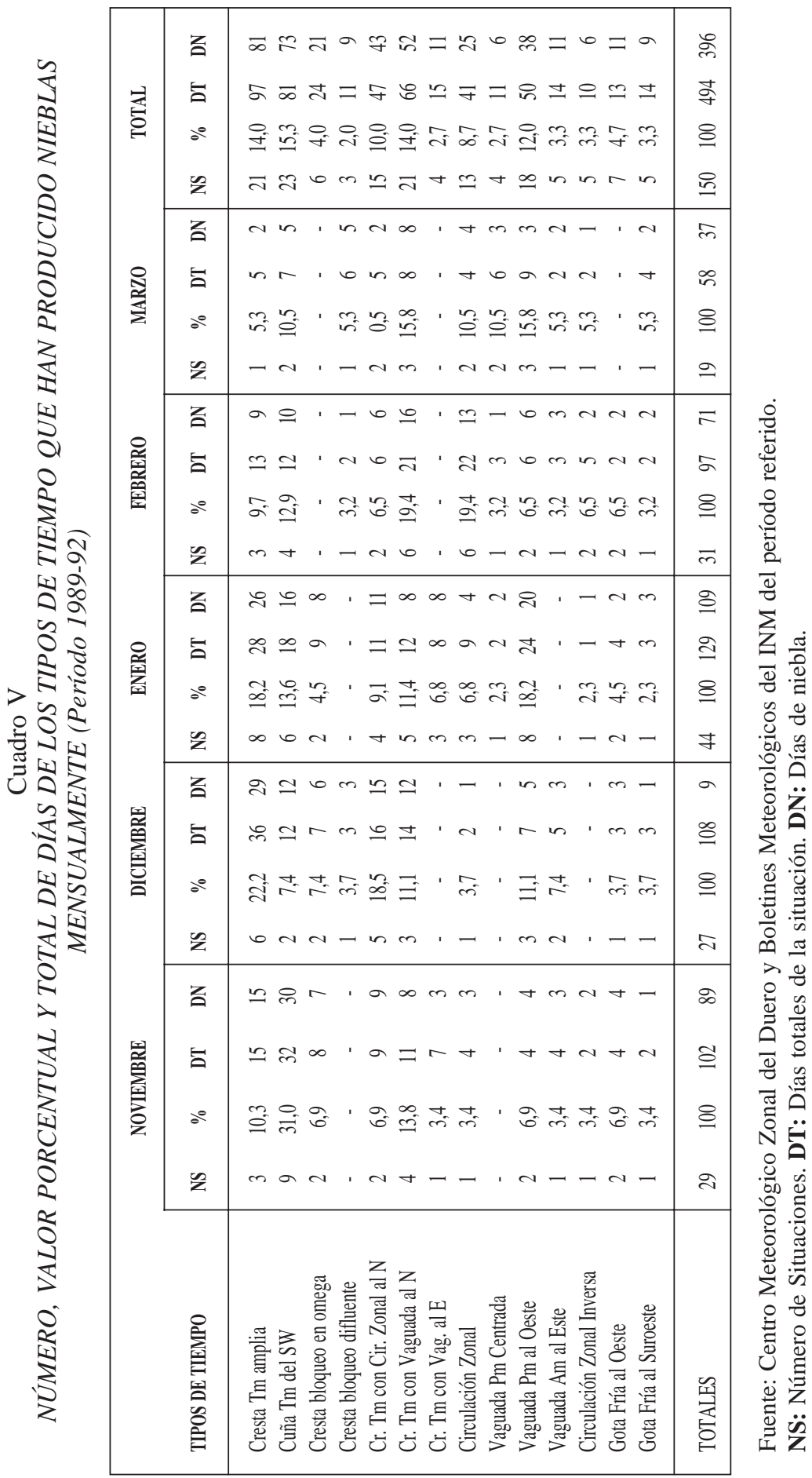


días de su evolución, pero no siempre con igual intensidad. Unas veces generan nieblas matinales que en el transcurso del día terminan por disipar. Por lo general se mantienen hasta mediodía y a más tardar hasta las 13 h., y en su levantar el denso velo de gotitas se condensa en distintas formaciones nubosas que suelen terminar por desaparecer. Su génesis se debe a fenómenos de irradiación nocturna, es decir, al fuerte enfriamiento de las noches con temperaturas próximas o por debajo de $0^{\circ} \mathrm{C}$, que genera en la capa más baja de la atmósfera procesos de inversión térmica, visibles en los amaneceres al condensarse el vapor de agua del aire en una capa de importante espesor. La niebla se apelmaza contra el valle, pero en los páramos y oteros luce el sol, con lo que se puede deducir un espesor en torno a los $100-150 \mathrm{~m}$, a veces más.

Con estas situaciones y dependiendo del mes del invierno en que nos encontremos, las temperaturas máximas diarias se aproximan a $\operatorname{los} 10^{\circ} \mathrm{C}$, aunque en los meses de comienzo y fin del invierno el calentamiento de mediodía es mucho más intenso alcanzándose los $15^{\circ} \mathrm{C}$. También se reduce el número de horas de niebla y su disipación se adelanta. Las temperaturas mínimas, no obstante, son bastante bajas, con valores por debajo de $\operatorname{los} 3-5^{\circ} \mathrm{C}$ en general, y con heladas muy frecuentes. Esto permite la aparición de escarchas, cubriendo el suelo, que en el transcurrir del día acaban desapareciendo.

Otras veces las nieblas son incapaces de levantar a lo largo de todo el día, manteniéndose esta situación durante varias jornadas consecutivas. Son las llamadas nieblas persistentes, debidas en este caso a fenómenos de advección-irradiación. Se originan cuando el aire tropical, cálido y húmedo, irrumpe sobre este espacio mucho más frío, al haberse enfriado en días previos por otras situaciones dinámicas. El contacto del aire Tm con una superficie a baja temperatura motiva condensaciones generalizadas en todo el valle, que logran mantenerse por largo tiempo ante el importante espesor que alcanzan. En realidad, la niebla se desarrolla a base de bancos más o menos continuos que ahora no sólo aparecen en el fondo del valle sino que logran remontar los páramos y extenderse hasta las plataformas sorianas. Es a partir de los $1.100 \mathrm{~m}$ cuando dejan de manifestarse. Lógicamente en estas últimas superficies no adquieren la entidad ni el mantenimiento que en los valles, donde con facilidad superan los 250-300 m de espesor. Estas son nieblas de advección al deberse a la irrupción de masas de aire más cálidas sobre un espacio muy frío, pero también lo son de irradiación pues en días sucesivos la falta de insolación se incrementa siendo importante las pérdidas de calor, con lo que las temperaturas adquieren valores más extremos.

Efectivamente, la falta de calentamiento continuado enfría cada vez más el ambiente experimentando las temperaturas drásticos descensos de forma progresiva. Es entonces cuando las máximas difícilmente alcanzan $\operatorname{los} 5^{\circ} \mathrm{C}$, pudiendo en algunas de sus jornadas no rebasar $\operatorname{los} 3^{\circ} \mathrm{C}$ o incluso $\operatorname{los} 0^{\circ} \mathrm{C}$; y las mínimas siempre ofrecen valores negativos siendo habituales descensos por debajo de los $-5^{\circ} \mathrm{C}$. Tampoco son desconocidas las jornadas en que tanto las máximas como las mínimas mantienen valores negativos.

Durante el mes de diciembre del año 1988 y gran parte de enero de 1989, las nieblas fueron un elemento muy generalizado. El dominio de situaciones anticiclónicas, con distintos tipos de circulaciones, en ambos meses fue su protagonista aunque no alcanzaron igual intensidad en todo ese tiempo. Del 22 de diciembre al 5 de enero, se volvieron persistentes a lo largo de todo el valle, no levantando en el transcurso del día y registrándose unos valores térmicos realmente crudos. A modo de ejemplo, en Valladolid la máxima más elevada en esos 15 días fue de $6,8^{\circ} \mathrm{C}$ y la más baja de $-1^{\circ} \mathrm{C}$, siendo en siete días inferiores a $3^{\circ} \mathrm{C}$. Por su parte, las mínimas estuvieron siempre por debajo de $2,6^{\circ} \mathrm{C}$ registrándose como valor más extremo $-7,5^{\circ} \mathrm{C}$, siendo en trece días negativas.

Con este tipo de situaciones es frecuente, por la densidad de la nubosidad creada, que aparezcan precipitaciones muy débiles, que rara vez superan el calificativo de inapreciable 
(como mucho alcanzan $1 \mathrm{~mm}$ ), pero que contribuyen a hacer más penoso el tiempo reinante. Siguiendo el mismo ejemplo anterior, en Valladolid durante cinco días se contabilizó este meteoro, no superando en ningún momento los $0,4 \mathrm{~mm}$. Como consecuencia esta niebla, llamada «llorona», termina helándose dados los bajos registros térmicos, apareciendo las cencelladas. Esta densa película de hielo que cubre el suelo y los objetos, asemejando en apariencia una nevada, se mantiene de un día para otro ante la falta de una insolación eficaz.

Continuando con el mismo caso de Valladolid, y computando sólo las situaciones antciclónicas que han deparado nieblas, un total de 149 días en los cinco años, se han registrado precipitaciones débiles en 59 días, siendo en su mayoría inferiores a $0,5 \mathrm{~mm}$ $(24,2 \%)$, e inapreciables $(6,7 \%)$. Cantidades superiores a un $\mathrm{mm}$ son realmente infrecuentes $(2 \%)$.

El rigor del frío, la elevada humedad ambiente, la falta de visibilidad hacen de este uno de los tipos de tiempo más desagradables del invierno, aunque singulares.

\section{b) Las nieblas con situaciones ciclónicas}

Entre las situaciones inestables capaces de provocar nieblas, nuevamente puede marcarse otra distinción por meses. Durante el trimestre noviembre-enero son las vaguadas Pm al oeste de la Península Ibérica las que las originan en mayor medida, sobre todo en diciembre y enero. En los meses de finales del invierno es la circulación zonal y las vaguadas Pm centradas las que toman el relevo. El resto de los tipos de tiempo tienen un grado de participación muchas veces testimonial al haberse computado tan sólo una situación. Esto, si a nivel estadístico no es relevante, si es indicativo del grado de versatilidad que tiene este meteoro en su génesis (cuadro V).

Estas nieblas en cualquier caso son de advección al formarse siempre por la irrupción de un aire más templado y húmedo que el existente por el lugar que se desplazan. En el valle del Duero, por lo general con temperaturas muy bajas durante el invierno, la llegada de aire polar marino motiva, ocasionalmente, inevitables procesos de condensación en las capas más bajas, dado el brusco contraste al que se ve sometido al arribar a suelo castellano. Sin embargo, en relación con estas nieblas, cabe establecer una serie de precisiones.

En primer lugar, dentro de las situaciones inestables capaces de provocarlas, son las asociadas al aire Pm con una entrada sobre la Península Ibérica occidental o suroccidental las que las ocasionan en mayor medida. En segundo lugar, en este tipo de situaciones la creación de nieblas no es un atributo dominante. De hacerlo, lo normal es que tan sólo aparezcan durante el primer día de su situación y en ocasiones tan sólo durante algunas horas, al ser la lluvia el elemento que realmente las caracteriza. En tercer lugar, sólo en contadas situaciones de vaguadas al oeste peninsular o de circulación zonal han logrado generarlas durante varios días seguidos (dos o tres). En este caso la razón hay que buscarla en los aportes tan cálidos que supone el aire Pm dada su procedencia y componente de entrada, oeste o suroeste, en relación a este espacio de marcado aislamiento.

Este mismo mecanismo es el que explica las nieblas con situaciones de gota fría. Si reparamos sólo han aparecido nieblas con aquéllas que permiten flujos cálidos y húmedos del suroeste (Cuadro V). Con ellas, dada la inestabilidad que las define, no es normal que se produzcan.

Otra situación en la que aparecen nieblas matinales, característica de los meses finales del invierno, sobre todo de marzo, es que tras la gestación de una tormenta, por la irrupción de una vaguada sobre un suelo en este caso recalentado (varios días de elevadas temperaturas), al día siguiente aparecen neblinas de escasa duración aprovechando los elevados 


\section{Cuadro VI}

NÚMERO DE DÍAS DE NIEBLA DE LAS DISTINTAS SITUACIONES DINÁMICAS POR MESES (Período 1988-92)

\begin{tabular}{|lrrrrrrrrrrrr|r|r|}
\hline & NOVIEMBRE & DICIEMBRE & ENERO & FEBRERO & MARZO & AÑ \\
\hline SITUACIón DINÁMICA & D & $\%$ & $\mathbf{D}$ & $\%$ & $\mathbf{D}$ & $\%$ & $\mathbf{D}$ & $\%$ & $\mathbf{D}$ & $\%$ & D & $\%$ \\
\hline Circulación Sur-Norte & 72 & 80,9 & 77 & 85,6 & 77 & 70,6 & 42 & 59,2 & 22 & 59,5 & 290 & 73,2 \\
Circulación Norte-Sur & 7 & 7,9 & 8 & 8,9 & 22 & 20,2 & 10 & 14,1 & 8 & 21,6 & 55 & 13,9 \\
Circulación Zonal & 3 & 3,4 & 1 & 1,1 & 4 & 3,7 & 13 & 18,3 & 4 & 10,8 & 25 & 6,3 \\
Circulación Zonal Inversa & 2 & 2,2 & 0 & 0,0 & 1 & 0,9 & 2 & 2,8 & 1 & 2,7 & 6 & 1,5 \\
Gotas Frías & 5 & 5,6 & 4 & 4,4 & 5 & 4,6 & 4 & 5,6 & 2 & 5,4 & 20 & 5,1 \\
\hline TOTAL & 89 & 100 & 90 & 100 & 109 & 100 & 71 & 100 & 37 & 100 & 396 & 100 \\
\hline
\end{tabular}

Fuente: Centro Meteorológico Zonal del Duero y Boletines Meteorológicos diarios del período referido.

registros de humedad ambiental. Este tipo de nieblas, no obstante, es más propio de la primavera-verano.

Las nieblas de advección no tienen la entidad ni desarrollo de las de génesis anticiclónica, pero también contribuyen a caracterizar las del valle medio del Duero. De un modo explícito puede observarse si se tiene en cuenta el porcentaje de días de niebla ocupado por las distintas situaciones dinámicas (Cuadro VI). El mayor peso de las de carácter anticiclónico es evidente, pues el 73,2 \% de los días de niebla se han debido a ellas, frente el 26,8 $\%$ producidas por dinámicas inestables (circulación norte-sur, circulación zonal, circulación inversa y gotas frías).

2. La proporción de nieblas en el valle medio del Duero: clasificación según su tipología

A su vez, este análisis nos ha permitido saber el número de nieblas habido en el valle medio del Duero durante este período de cinco años y, poder suponer las que pueden afectarle como promedio a lo largo del invierno. No se trata nada más que computar en un único valor, el del valle, todos aquellos días en que ha habido niebla en algún punto del mismo. Se pretende computar el valor medio que se puede producir en el mismo a lo largo del invierno, que lógicamente no puede coincidir con la media de cada observatorio concreto dado que las nieblas no siempre tienen un carácter general.

Así, se explican los casi 85 días del mismo (Cuadro VII), que a su vez aparecen en la figura 5 como valor porcentual sobre el total. Dicho número supone el 56,2\% de los cinco

Cuadro VII

PROPORCIÓN DE DÍAS DE NIEBLA INVERNALES EN EL VALLE MEDIO DEL DUERO (Período 1988-92)

\begin{tabular}{|ccccccc|}
\hline & Nov. & Dic. & Enero & Febr. & Marzo & TOTAL \\
\hline Valor medio & 18,8 & 21,0 & 22,4 & 15,0 & 7,6 & 84,8 \\
\hline
\end{tabular}

Fuente: Centro Meteorológico Zonal del Duero. Datos diarios. 
Días SIN niebla $43,8 \%$
Días CON niebla

$56,2 \%$

ENERO 26,4\%

FEBRERO $17,6 \%$

MARZO $9,0 \%$

TOTAL PERÍODO INVERNAL

Desglose mensual

FIGURA 5. Proporción mensual de días de niebla en el valle medio del Duero. Período 1988-92.

CUADRO VIII

PORCENTAJE DE LOS DISTINTOS TIPOS DE NIEBLA EN EL VALLE MEDIO DEL DUERO SEGÚN SUS CARACTERÍSTICAS

\begin{tabular}{|c|c|c|c|c|c|}
\hline \multicolumn{6}{|c|}{ Situaciones Anticiclónicas } \\
\hline & \multicolumn{2}{|c|}{ Distribución espacial } & \multicolumn{2}{|c|}{ Duración temporal } & \multirow[t]{2}{*}{ Tipo } \\
\hline & Generalizada & Aislada & Sit. >1 día & Sit. 1 día & \\
\hline Matinal & 25,5 & 22,7 & 45,5 & 3,0 & Irradiación \\
\hline Persistente & 20,5 & 4,5 & 24,5 & 0,2 & Advección-Irradiación \\
\hline \multicolumn{6}{|c|}{ Situaciones Ciclónicas } \\
\hline & \multicolumn{2}{|c|}{ Distribución espacial } & \multicolumn{2}{|c|}{ Duración } & \multirow[t]{2}{*}{ Tipo } \\
\hline & Generalizada & Aislada & Sit. >1 día & Sit. 1 día & \\
\hline Matinal & 2,5 & 16,2 & 14,4 & 4,8 & Advección \\
\hline Persistente & 2,8 & 5,3 & 6,1 & 1,5 & Advección \\
\hline
\end{tabular}

Fuente: Boletines Meteorológicos diarios del período 1988-92 y datos diarios de niebla del período referido en los observatorios analizados. Centro Meteorológico Zonal del Duero.

meses de invierno (151 días), y el 67,2\% del trimestre noviembre-enero (92 días). Conviene advertir que tan elevado índice es posible dado que define al de todo el valle analizado, y además se ha comprobado que dicho número no se debe a situaciones distintas registradas en puntos dispersos del valle, sino al complemento de una dada. Es decir, que una misma situación no origina los mismos días de niebla en distintas partes del valle, pero las de todos ellos se complementan porque responden a la misma dinámica atmosférica que se mantiene durante varios días.

En un intento de hacer una clasificación sobre la distinta tipología de las nieblas en el valle medio del Duero se ha elaborado un cuadro que sintetiza sus posibles manifestaciones, jugando con las variables espacial y temporal en la forma de manifestarse. Igualmente, se ha especificado la procedencia dinámica de las mismas (Cuadro VIII). 
Las situaciones anticiclónicas son las que producen nieblas de mayor extensión y espesor, generalizadas para todo el valle, el $46 \%$ frente al 27,2\% de las de carácter aislado. Dentro de aquéllas son las matinales las dominantes (el 25,5\%), aunque las persistentes también tienen gran protagonismo (el 20,5\%). En cualquier caso, sean dispersas o afecten en mayor extensión, dominan las nieblas de irradiación por fenómenos de inversión sobre las de advección-irradiación (el 48,2\% frente al 25\% respectivamente). Además, las nieblas de situaciones anticiclónicas se desarrollan a lo largo de varios días por lo general, con independencia de su grado de intensidad, siendo realmente escasas, el 3,2\%, aquellas que tan sólo aparecen en un solo día.

El comportamiento de las nieblas ciclónicas en cierto modo es contrario. Dominan desde un punto de vista espacial las de tipo localizado (el 21,5\% frente al 5,3\% de las generalizadas), mientras que temporalmente tienen mayor peso las que son breves en duración e intensidad (Cuadro VIII).

\section{La influencia espacial de las nieblas según los tipos de tiempo}

Otro aspecto a destacar es ver el desarrollo espacial que tienen las nieblas con cada tipo de tiempo. A este respecto en cada una de las situaciones que han originado nieblas y según tipos de tiempo, se ha especificado si aparecían de forma generalizada en todo el valle ${ }^{2}$, si lo hacían en su mitad occidental, es decir, hasta Aranda de Duero, si lo hacían en su mitad oriental (cuenca satélite propiamente dicha), o si aparecían con total aleatoriedad y de forma aislada.

De este modo, se ha puesto de manifiesto que con las crestas Tm amplias y con aquellas que quedan flanqueadas por el norte por circulaciones zonales, que siempre originan inestabilidad en el Cantábrico, en más del $70 \%$ de las casos han generado niebla a lo largo de todo el valle, llegándose a sobrepasar el $75 \%$ con las cuñas Tm de penetración suroeste (Cuadro IX). Porcentajes también elevados de nieblas generalizadas producen las situaciones de bloqueo en omega y las crestas con vaguadas al norte, con $57,1 \%$ y $40,4 \%$ respectivamente.

En cambio, el que la niebla sea un meteoro localizado y reducido a un solo día es habitual con las dinámicas más inestables, es decir, circulaciones zonales (88\%), vaguadas Pm centradas $(100 \%)$ o localizadas al oeste $(47,4 \%)$, circulaciones inversas $(66,7 \%)$, colada Am al este $(90,9 \%)$ y gotas frías al oeste $(81,8 \%)$ y suroeste $(55,6 \%)$, aunque también es característico de los bloqueos difluentes (77,8\%). Es importante relacionar estos porcentajes con los números de días por ellas ocupados para darnos cuenta de la escasa representación que en conjunto tienen, si bien algunos de ellos (vaguadas Pm al oeste y la circulación zonal) son significativos (Cuadro IX).

En el caso concreto de las vaguadas Pm al oeste peninsular es importante destacar que, en ocasiones, generan nieblas generalizadas a lo largo del valle (42,1\% de las ocasiones). Esto siempre ha ocurrido si con anterioridad a la irrupción del aire polar existía sobre suelo castellano aire muy frío y estable donde las heladas nocturnas estaban aseguradas.

Con determinados tipos de tiempo a veces la niebla sólo se ha registrado en la mitad occidental u oriental del valle. Son más hechos puntuales que comportamientos generalizables. Únicamente con las crestas con vaguadas al norte o al este de España han llegado a suponer porcentajes por encima del $25 \%$ y en ambos casos para la mitad oriental.

2 Conviene apuntar que por «niebla generalizada» se ha considerado aquella que se ha computado en distintos puntos del valle al mismo tiempo, homogéneamente distribuidos y siempre que se diera en un mínimo de 10 observatorios. 
Cuadro IX

PORCENTAJE DEL NÚMERO MEDIO DE DÍAS DE NIEBLA EN CADA TIPO DE

TIEMPO

\begin{tabular}{|lcccrrr|}
\hline TIPOS DE TIEMPO & $\begin{array}{c}\text { Niebla } \\
\text { Generaliz. }\end{array}$ & $\begin{array}{c}\text { Mitad } \\
\text { Oeste }\end{array}$ & $\begin{array}{c}\text { Mitad } \\
\text { Este }\end{array}$ & $\begin{array}{c}\text { Niebla } \\
\text { Aislada }\end{array}$ & $\begin{array}{c}\mathbf{N}^{\circ} \text { Días } \\
\text { de Niebla }\end{array}$ & $\begin{array}{c}\text { \% } \\
\text { Días }\end{array}$ \\
\hline Cresta Tm amplia & 71,6 & 4,9 & 4,9 & 18,5 & 81 & 20,5 \\
Cuña Tm del SW & 76,7 & 2,7 & 1,4 & 19,2 & 73 & 18,4 \\
Cresta bloqueo en omega & 57,1 & 14,3 & 23,8 & 4,8 & 21 & 5,3 \\
Cresta bloqueo difluente & 22,2 & - & - & 77,8 & 9 & 2,3 \\
Cr. Tm con Cir. Zonal al N & 72,1 & 7,0 & 4,7 & 16,3 & 43 & 10,9 \\
Cr. Tm con Vaguada al N & 40,4 & - & 25,0 & 34,6 & 52 & 13,1 \\
Cr. Tm con Vag. al E & 27,3 & - & 27,3 & 45,5 & 11 & 2,8 \\
Circulación Zonal & 8,0 & - & 4,0 & 88,0 & 25 & 6,3 \\
Vaguada Pm Centrada & - & - & - & 100,0 & 6 & 1,5 \\
Vaguada Pm al Oeste & 42,1 & - & 10,5 & 47,4 & 38 & 9,6 \\
Vaguada Am al Este & - & - & 9,1 & 90,9 & 11 & 2,8 \\
Circulación Zonal Inversa & 33,3 & - & - & 66,7 & 6 & 1,5 \\
Gota Fría al Oeste & - & - & 18,2 & 81,8 & 11 & 2,8 \\
Gota Fría al Suroeste & 33,3 & 11,1 & - & 55,6 & 9 & 2,3 \\
\hline M E D I A & 52,0 & 3,3 & 9,1 & 35,6 & 396 & 100 \\
\hline
\end{tabular}

Fte: Centro Meteorológico Zonal del Duero. Datos diarios.

Por lo tanto, queda puesto de manifiesto la hegemonía de las situaciones anticiclónicas a la hora de producir nieblas, hecho evidente, pero ahora sabemos la proporción de días en que hay nieblas con cada una de ellas, la posibilidad de que aparezcan con otros tipos de tiempo y el carácter que las definen.

\section{Los efectos más inmediatos de las nieblas}

Por último, cabría destacar las consecuencias que se derivan de las nieblas, tanto a nivel perceptivo como sobre hechos concretos. Aspecto que necesitaría un análisis mucho más desarrollado, pero del que sólo se pretende apuntar algunas pinceladas.

Si se hace un balance de las nieblas y sobre todo de éstas en relación con el hombre, son más elementos negativos que positivos los que de ellas se derivan. Varios hechos lo atestiguan. En primer lugar, conllevan una importante reducción de la visibilidad, con el peligro que esto supone para el tráfico rodado en general. Condicionan el uso que se haga de los transportes, pues en situaciones de nieblas intensas se reduce el número de automóviles en circulación, y aumenta la prudencia en la conducción. La menor velocidad a la que se circula es ya un importante elemento reductor del número de accidentes. No obstante, es obvio que también es uno de sus principales responsables, principalmente en carretera.

Conclusiones a las que se ha llegado tras analizar el número de accidentes con víctimas habido en el período 1988-92, en la carretera N-122, entre Valladolid y su límite de 
provincia más oriental ${ }^{3}$. En esos cinco años, en los meses de noviembre a marzo, se produjeron un total de 106 accidentes. El máximo mensual recayó en diciembre en estrecha vinculación con el período vacacional ( 28 accidentes). Todos ellos depararon un total de 12 muertos y 187 heridos. En más de la mitad de estos accidentes, en concreto en el 54,7\%, la niebla estuvo presente. Es decir, que en esos días la niebla era un elemento más que pudo contribuir a su producción. Sin embargo, sólo se señala explícitamente a la niebla como principal responsable del accidente en 23 casos, el 21,7\%. Valores que confirman que la niebla, frecuente en este valle, se convierte en un factor social de gran trascendencia.

También negativa es su contribución en los procesos de contaminación ambiental, especialmente sensibles en la ciudad de Valladolid. Dadas las bajas temperaturas invernales y su coincidencia en numerosas ocasiones con períodos de niebla, la necesidad en el uso de calefacciones es mayor, con lo que el aporte de partículas y gases contaminantes aumenta (sobre todo los derivados del azufre y de los hidrocarburos), en una atmósfera con escasa capacidad de disipación. A este respecto habría que añadir el cada vez mayor parque automovilístico. Así mismo, contribuyen los aportes de actividades industriales, localizadas en el entorno de la ciudad, con emisiones de derivados del nitrógeno que repercuten en un descenso de la calidad del aire. De todo ello, se puede deducir que si no causa directa, sí contribuyen a un incremento de las enfermedades respiratorias.

Otro factor de menor transcendencia y frecuencia, es el aumento del riesgo de accidentes en los transeúntes en situaciones de cencellada, al encontrarse el suelo helado. Pero más importancia tiene un factor de carácter social que es derivado de los problemas de delincuencia ante la falta de visibilidad (robos, violaciones...).

Pero a su vez, cabe señalar algunos efectos positivos, aunque principalmente fuera de los ámbitos urbanos. La frecuencia de nieblas introduce una mayor humedad en las tierras de cultivo, suponiendo un aporte hídrico, aunque de difícil estimación. Al producirse en el invierno no dificultan el desarrollo de las plantas por no coincidir con el período de eclosión vegetal. Como consecuencia de las bajas temperaturas que las nieblas se encargan de prolongar, se provocan frecuentes escarchas y cencelladas que al deshelarse suponen otro aporte más de agua a la tierra.

\section{Conclusiones}

A medida que nos acercamos a la cabecera del Duero, la intensidad de los procesos neblinosos lejos de disminuir, como cabría esperar, mantienen sus índices cuando no los aumentan. Su ubicación más oriental se compensa al manifestarse de forma más acendrada los condicionantes locales, reafirmando la hipótesis de que junto a la dinámica atmosférica, son las condiciones de la Cuenca, y en su caso la del Burgo de Osma-Almazán, factores de primera índole al caracterizar este meteoro.

Así pues, son las condiciones de aislamiento, morfológicas y altitudinales las que favorecen la formación de nieblas y las que las caracterizan en su forma de presentarse, en su persistencia e intensidad.

Pero junto a las condiciones geomorfológicas, es la dinámica atmosférica la que se encarga de discernir entre distintos tipos de nieblas y frecuencias.

3 Este análisis sólo se ha realizado de aquellos accidentes que han deparado víctimas, bien fueran mortales o heridos. No se computan accidentes sólo con daños materiales, dado que únicamente se da parte de ellos cuando no hay entendimiento entre los implicados y se requiere levantar atestado. No obstante, éstos son mucho más numerosos, y si bien no se trata de accidentes graves si son pequeñas colisiones (sobre todo en el ámbito urbano), salidas de la carretera y alcances muchas veces motivados por poca visibilidad. 
Se ha constatado la mayor importancia de las nieblas producidas en situaciones anticiclónicas y su diferente comportamiento según distintos tipos de circulación. Así, unas veces aparecen nieblas matinales de irradiación, y otras, las más persistentes de advecciónirradiación. También se ha comprobado el menor peso, aunque no por ello menos importante, de las nieblas de advección provocadas en situaciones inestables. Todas ellas definen uno de los rasgos climáticos más característicos de los inviernos del valle del Duero, donde se registra un promedio muy elevado, con casi ochenta y cinco días.

La niebla, se convierte así en un aspecto propio del clima de este valle, creando un ambiente singular, identificador de la particular crudeza y rigor de sus inviernos.

\section{Fuentes y bibliografía}

\section{Fuentes}

\section{CENTRO METEOROLÓGICO ZONAL DEL DUERO}

Datos diarios de días de niebla para la serie de años de que disponen los observatorios reflejados en los cuadros.

Datos diarios de precipitación para la serie de años de que disponen los observatorios señalados.

Datos diarios de temperatura del período comprendido entre 1988-92 en los observatorios señalados.

\section{SERVICIO METEOROLÓGICO NACIONAL}

Boletines Diarios y Resúmenes mensuales del período 1988-92.

\section{JEFATURA PROVINCIAL DE TRÁFICO DE VALLADOLID}

Partes de accidentes de circulación con víctimas en la N-122 en el período 1988-92 (noviembremarzo).

\section{Bibliografía}

CALONGE CANO, G. (1984): Climatología de los inviernos de Valladolid. Publicaciones de la Universidad de Valladolid. Valladolid. $357 \mathrm{pp}$.

GARCÍA DE PEDRAZA, L. \& GARCÍA SANJUAN, J. (1978): Diez temas sobre el clima. $\mathrm{M}^{\circ}$ de Agricultura. Madrid. 213 pp.

GARCÍA FERNÁNDEZ, J. (1986): El clima en Castilla y León. Ed. Ámbito.Valladolid. 370 pp.

HERNÁNDEZ, M.A.L. \& DEL VALLE, J. (1989): «Las nieblas en la depresión del Ebro: el caso del Campo de Zaragoza y la Hoya de Huesca». Geographicalia. 26. Zaragoza, pp. 125-139.

ORTEGA VILLAZÁN, M.T. (1992): El Clima del sector norte de la Cordillera Ibérica. Secretariado de Publicaciones de la Universidad de Valladolid. Valladolid. 359 pp.

PUIGDEFABREGAS TOMÁS, J. (1970): «Características de la inversión térmica en el extremo oriental de la depresión interior altoaragonesa». Pirineos. 96. Jaca, pp. 21-47. 